\title{
Transcranial direct current stimulation of the primary motor cortex improves word-retrieval in older adults
}

\section{Marcus Meinzer ${ }^{1,2}$, Robert Lindenberg ${ }^{2}$, Mira M. Sieg ${ }^{2}$, Laura Nachtigall ${ }^{2}$, Lena UIm ${ }^{1,2}$ and Agnes Flöel ${ }^{2,3 *}$}

\author{
${ }^{1}$ Center for Clinical Research, The University of Queensland, Herston, QLD, Australia \\ 2 Department of Neurology, NeuroCure Clinical Research Center, Charité University Medicine, Berlin, Germany \\ ${ }^{3}$ Department of Neurology, Center for Stroke Research Berlin, Charité University Medicine, Berlin, Germany
}

\section{Edited by:}

David Bartrés-Faz, University of Barcelona, Spain

Reviewed by:

Lutz Jäncke, University of Zurich,

Switzerland

Roy H. Hamilton, University of

Pennsylvania, USA

\section{*Correspondence:}

Marcus Meinzer, Centre for Clinical

Research, The University of

Queensland, Building 71/918, Royal

Brisbane \& Women's Hospital

Campus, Herston, QLD 4029,

Australia

e-mail:m.meinzer@uq.edu.au; Agnes Flöel, Department of

Neurology, Center for Stroke Research Berlin and NeuroCure Clinical Research Center, Charité University Medicine, Charitéplatz 1 10117 Berlin, Germany

e-mail: agnes.floeel@charite.de
Language facilitation by transcranial direct current stimulation (tDCS) in healthy individuals has generated hope that tDCS may also allow improving language impairment after stroke (aphasia). However, current stimulation protocols have yielded variable results and may require identification of residual language cortex using functional magnetic resonance imaging (fMRI), which complicates incorporation into clinical practice. Based on previous behavioral studies that demonstrated improved language processing by motor system pre-activation, the present study assessed whether tDCS administered to the primary motor cortex (M1) can enhance language functions. This proof-of-concept study employed a sham-tDCS controlled, cross-over, within-subject design and assessed the impact of unilateral excitatory (anodal) and bihemispheric (dual) tDCS in 18 healthy older adults during semantic word-retrieval and motor speech tasks. Simultaneous fMRI scrutinized the neural mechanisms underlying tDCS effects. Both active tDCS conditions significantly improved word-retrieval compared to sham-tDCS. The direct comparison of activity elicited by word-retrieval vs. motor-speech trials revealed bilateral frontal activity increases during both anodal- and dual-tDCS compared to sham-tDCS. This effect was driven by more pronounced deactivation of frontal regions during the motor-speech task, while activity during word-retrieval trials was unaffected by the stimulation. No effects were found in $\mathrm{M} 1$ and secondary motor regions. Our results show that tDCS administered to M1 can improve word-retrieval in healthy individuals, thereby providing a rationale to explore whether M1-tDCS may offer a novel approach to improve language functions in aphasia. Functional magnetic resonance imaging revealed neural facilitation specifically during motor speech trials, which may have reduced switching costs between the overlapping neural systems for lexical retrieval and speech processing, thereby resulting in improved performance.

Keywords: transcranial direct current stimulation, functional magnetic resonance imaging, language, motor, aging

\section{INTRODUCTION}

The language and motor action systems feature tight functional connections and share neural resources (Willems and Hagoort, 2007). They are organized in partially overlapping neural networks where higher order cortices can be involved in a flexible, context-dependent manner in different functions (Bressler and Menon, 2010; Behrens and Sporns, 2012). In the context of language production, the cortico-bulbar system controls muscles involved in speech and breathing. Furthermore, cortico-subcortical loops comprising primary (M1) and non-primary motor areas as well as the ventrolateral thalamus and striatum are not only involved in the initiation and sequencing of speech, but also in cognitive control processes (Crosson, 2013; Dick et al., 2013). Besides reciprocal corticosubcortical interplay, cortical motor areas are strongly connected with inferior frontal regions that are crucial for word-retrieval processes and also the selection of motor actions (ThompsonSchill et al., 1997; Pobric and Hamilton, 2006; Eickhoff et al., 2009).

While there is an on-going debate about the origin and extent of language-motor system interactions and also the (linguistic) level at which the motor system impacts on language processing, the mutual interplay between the two systems is generally not questioned (Rizzolatti and Craighero, 2004; Willems and Hagoort, 2007; Pulvermuller and Fadiga, 2010). The behavioral relevance of these interactions is illustrated by studies showing that lexical retrieval and semantic processing can be facilitated by execution or observation of manual gestures (Hadar et al., 1998; Holle and Gunter, 2007; Dick et al., 2009) and prohibiting manual gestures can slow down speech production (Rauscher et al., 1996; Pine et al., 2007). Pre-activation of the motor system by different behavioral interventions also 
improved lexical retrieval in patients with post-stroke aphasia (Hanlon et al., 1990; Harnish et al., 2011; Meinzer et al., 2011a; Benjamin et al., 2014). So far, however, the neural mechanisms by which the motor system facilitates language production remain largely elusive.

Motor cortex excitability can be modulated by non-invasive transcranial direct current stimulation (tDCS; Stagg and Nitsche, 2011), providing a promising tool to alter interactions between motor and language systems. During tDCS, weak electrical currents are administered to the scalp that exert local effects on the underlying cortex, but also on functionally connected remote regions (Miniussi et al., 2013; Flöel, 2014). Excitatory tDCS (anodal-tDCS) facilitates motor learning when applied to M1 contralateral to the hand involved in a task (Reis et al., 2009) and these effects may be even more pronounced with simultaneous inhibitory (cathodal) tDCS to the ipsilateral M1 (dual-tDCS, (Vines et al., 2008)). In the language domain it has been shown that anodal-tDCS administered to perisylvian regions of the left language dominant hemisphere improved language processing (Flöel, 2012; Monti et al., 2013) and learning (de Vries et al., 2010; Meinzer et al., 2014a). Moreover, inhibitory tDCS of M1 impaired learning of a novel lexicon (Liuzzi et al., 2010). However, so far, it is unknown if facilitation of M1 by anodal-tDCS can improve linguistic processing.

Therefore, the present randomized, sham-controlled study employed a within-subjects design to study effects of M1 stimulation on semantic word-generation. Given that M1-tDCS has been suggested as an adjunct treatment approach for post-stroke motor (Lindenberg et al., 2010, 2012) and language rehabilitation (Pulvermüller and Berthier, 2008; Meinzer et al., 2011a), and that stroke typically affects older people, the present proofof-concept study assessed the impact of M1-tDCS in healthy older adults. We combined tDCS with simultaneous functional magnetic resonance imaging (fMRI) to assess its effects on task performance and brain activity (Meinzer et al., 2014b). Using the same task, we have previously demonstrated that anodal-tDCS administered to the left inferior frontal gyrus facilitated wordretrieval and selectively reduced task-related activity in bilateral prefrontal cortices during word-retrieval, but not during a simple motor speech baseline task (Meinzer et al., 2013). However, given that the exact (linguistic) level at which the motor system impacts on language processing is still under scrutiny (Willems and Hagoort, 2007) and that M1 stimulation may affect functional brain activity in premotor and prefrontal regions (Antal et al., 2011; Lindenberg et al., 2013), we explored whether potential tDCS effects on performance would be mediated by modulations of motor and/or language regions during either or both of these tasks.

\section{METHODS \\ STUDY OVERVIEW}

Data was acquired in the context of a study that assessed the impact of M1 stimulation on motor and linguistic performance and brain functions during resting-state and task-related fMRI. In a sham-tDCS controlled, within-subject, triple cross-over design, participants were scanned during three MRI sessions with simultaneous intrascanner tDCS. Active stimulation was administered during two of these sessions, either unilaterally to the left M1 (anodal-tDCS) or bilaterally (dual-tDCS). During dual-tDCS, anodal-tDCS was administered to the left M1, while cathodal-tDCS was administered simultaneously to the right M1 (Lindenberg et al., 2013).

During each session, participants first completed a restingstate scan followed by two subsequent task-related fMRI scans (an overt semantic word-retrieval task and a motor choice reaction task, Figure 1A illustrates the design of the study). The three scanning sessions were separated by approximately 1 week to prevent potential carry over effects of the active stimulation conditions and the order of stimulation conditions was counterbalanced across subjects. Please note, only data acquired during the semantic word generation task is reported here. The impact of the three stimulation conditions on resting-state functional connectivity and activity elicited by the motor choice reaction task have previously been reported (Lindenberg et al., 2013). The experimental set-up was identical to previous cross-over studies of our group that assessed the impact of anodal-tDCS administered to left perisylvian language areas on semantic wordretrieval (Meinzer et al., 2012a, 2013). The study was approved by the ethics committee of the Charite University Hospital, and conducted in accordance with the Helsinki declaration. Written informed consent was obtained from all subjects prior to study inclusion.

\section{SUBJECTS}

Eighteen healthy older adults participated in this study ( 9 women, 9 men; mean age $68.38 \pm 5.15$ years). Details of the sample have been reported previously (for details see Lindenberg et al., 2013 and Table 1). In short, all participants presented with age-appropriate cognitive status (as assessed by the test battery established by The Consortium to Establish a Registry for Alzheimer's Disease, CERAD-Plus ${ }^{1}$ ), and structural imaging parameters. They were right-handed according to the Edinburgh Handedness Inventory (mean \pm SD $91 \pm 15$, Oldfield, 1971), participated for the first time in a tDCS study, had no history of current or previous neurological or psychiatric disorders and did not report use of psychoactive medication or recreational drugs.

\section{TRANSCANIAL DIRECT CURRENT STIMULATION}

A constant direct current $(1 \mathrm{~mA})$ was administered by an MRI-compatible stimulator (DC-Stimulator Plus ${ }^{\circledR}$, NeuroConn, Ilmenau, Germany) using an established set-up (for details see Meinzer et al., 2014b). The anode was placed inside a $5 \times 7 \mathrm{~cm}^{2}$ saline-soaked sponge pocket and attached over the left M1 in all stimulation conditions (C3 of the 10-20 EEG system) as described in our previous manuscript (Lindenberg et al., 2013). For dual stimulation, the cathode $\left(5 \times 7 \mathrm{~cm}^{2}\right)$ was placed over the right M1 (position C4). During anodal-tDCS the reference electrode $\left(10 \times 10 \mathrm{~cm}^{2}\right)$ was positioned over the right supraorbital region. Those electrode montages represent the most commonly used set-ups for unihemispheric anodal-tDCS and dual-tDCS of the motor cortex. During sham-tDCS the reference electrode was pseudo-randomly assigned to either the right supraorbital region

\footnotetext{
${ }^{1} \mathrm{http}: / /$ www.memoryclinic.ch
} 


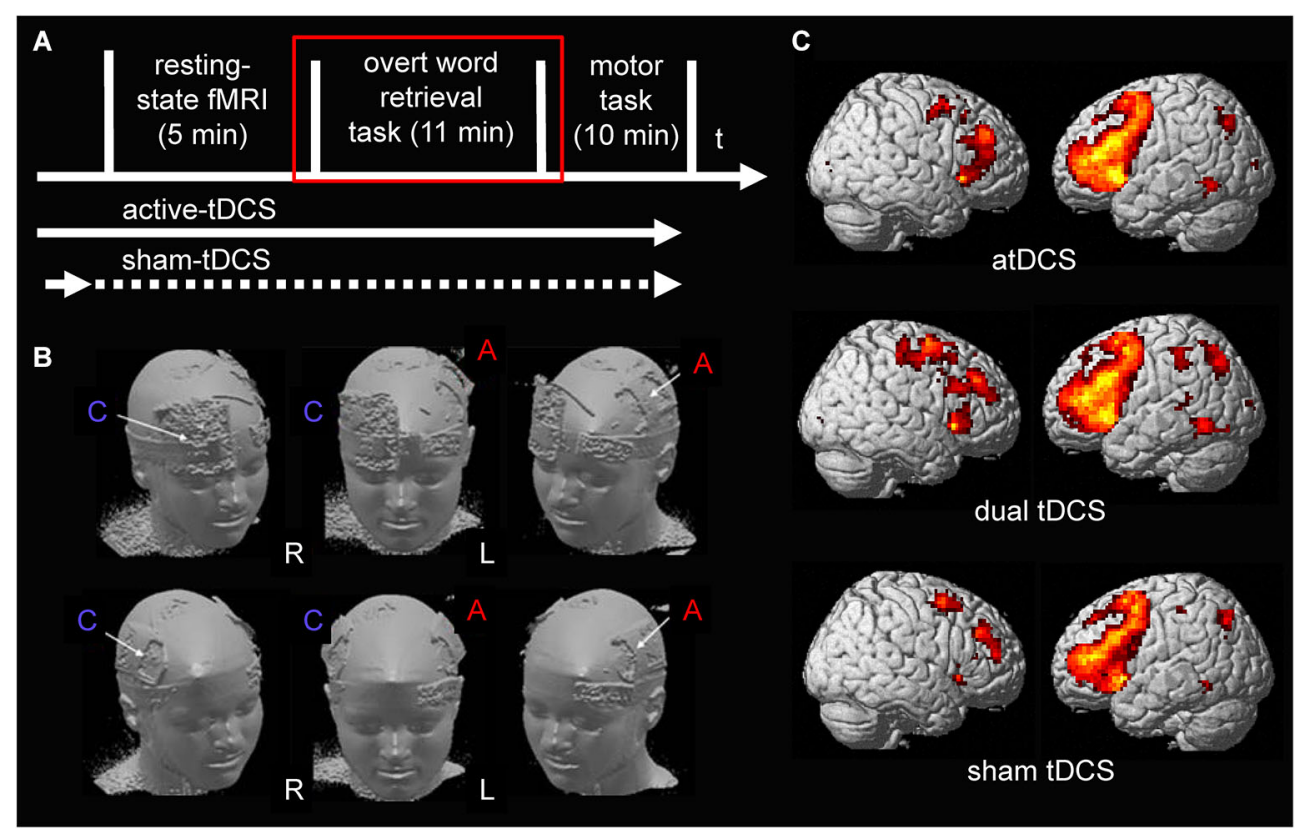

FIGURE 1 | (A) Illustrates the design of the present study. tDCS was ramped up prior to the start of the resting-state scan during all stimulation conditions. During sham-tDCS the current was ramped down after $30 \mathrm{~s}$ (before scanning commenced). During both active stimulation conditions (anodal- and dual-tDCS), it continued until after the end of the word-retrieval task (red square; only data acquired during the word-retrieval task is reported). (B) Surface rendering illustrates the location of the electrodes during the two active stimulation conditions on the scalp: upper row shows anodal-tDCS montage; lower row shows dual-tDCS montage. Please note, the larger size of the reference electrode (cathode) during anodal-tDCS renders the stimulation over the supraorbital cortex functionally ineffective. $\mathrm{R}=$ right hemisphere, $\mathrm{L}=$ left hemisphere. $\mathrm{C}=$ Cathode, $\mathrm{A}=$ Anode. (C) Surface rendering of activity patterns elicited by semantic word-retrieval vs. motor speech trials during the three stimulation conditions. Overall, as in previous studies that used the same task, this contrast elicited mainly activity in bilateral lateral frontal and medial frontal and premotor regions. Right column = Left hemisphere, Left column = Right hemisphere. All contrasts were thresholded at $p<0.05$, family-wise-error corrected at voxel and cluster levels. or right $\mathrm{M} 1$ in half of the participants to counterbalance those two montages across the group. Figure 1B illustrates the respective montages.

In all stimulation conditions, the current was initially increased to $1 \mathrm{~mA}$ in a ramp-like fashion over $10 \mathrm{~s}$ shortly prior to the start of the RS-sequence and remained constant for $30 \mathrm{~min}$ during anodal-tDCS and dual-tDCS, thereby covering the entire duration of the language task (which took approximately $11 \mathrm{~min}$, see below). During sham-tDCS, the current was turned off after $30 \mathrm{~s}$ prior to the start of the RS-sequence. In all stimulation conditions, the current was ramped down over $10 \mathrm{~s}$ at the end of the stimulation.

\section{fMRI TASK AND STIMULUS CHARACTERISTICS}

Magnetic resonance imaging data were acquired using a 3-Tesla Siemens Trio MR scanner at the Berlin Center for Advanced Neuroimaging (Charité University Hospital, Berlin, Germany). The overt semantic word-retrieval task was identical as in previous studies of our group (Meinzer et al., 2012a, 2013) and employed a T2*-weighted echo-planar imaging (EPI) sequence (TR/TA $=6000 / 2000 \mathrm{~ms}, \mathrm{TE}=30$, flip angle: $90^{\circ}, 32$ transverse slices, gap: $0.75 \mathrm{~mm}$, interleaved acquisition, FOV: $192 \times 192$, acquisition matrix: $64 \times 64,104$ volumes) and a temporal sparse sampling design. This allows assessing overt verbal responses during a scanner off phase to avoid articulation related artifacts. Six semantic categories ( 6 blocks of 10 consecutive trials of the same category, trial duration $3.8 \mathrm{~s}$ ) were presented using a projector and a system of mirrors. Participants were instructed to overtly produce one different exemplar during each trial or say "next" in case they could not come up with a response. In between trials, a black screen was displayed (2.2 s) and the hemodynamic response was acquired (sparse sampling). Task blocks alternated with a simple motor speech baseline condition (saying the word "rest"; five consecutive trials) in response to a written cue. Eighteen pre-selected semantic categories were used that were divided into three matched sets based on published norms ( Set $_{1}$ : trees, insects, sports equipment, body parts, beverages, occupations; Set $_{2}$ : flowers, fish, kitchen appliances, clothing, food, hobbies, Set $_{3}$ : spices, birds, toys, colors, auto parts, musical instruments; Sets $1 / 2 / 3$ : total \# exemplars produced in norm group: 1586/1587/1650, average category size: $11.6 / 11.8 / 12.2$; fluency: $0.64 / 0.60 / 0.59$, all $p>0.0 .92$; Mannhaupt, 1983). In addition, 20 different subjects participated in a pilot study that assessed performance using these 18 categories during a standard semantic verbal fluency task (duration: $1 \mathrm{~min}$; categories were presented in randomized order). The number of exemplars produced was comparable between the three sets (mean $\pm \mathrm{SD} \#$ correct 
Table 1 | Demographic characteristics of the participants and details of the neuropsychological assessment (mean and standard deviation, raw data).

\begin{tabular}{lcc}
\hline & Mean \pm SD & Range \\
\hline Age (years) & $68.38 \pm 5.15$ & $61-77$ \\
Education (years) & $15.88 \pm 4.74$ & $11-19$ \\
Sex (females/males) & $9 / 9$ & \\
CERAD subtests & & \\
Mini mental state examination & $29.44 \pm 0.62$ & $28-30$ \\
Verbal fluency & & \\
(\# examplars produced in one minute) & & \\
Semantic fluency & $25.61 \pm 9.41$ & $14-31$ \\
Phonemic fluency & $16.39 \pm 4.37$ & $12-25$ \\
Boston naming test & $14.67 \pm 0.59$ & $13-15$ \\
Verbal learning and memory & & \\
test (\# correct) & & \\
Learning success (sum score runs 1-3) & $20.44 \pm 3.55$ & $16-27$ \\
Delayed recall & $7.89 \pm 1.84$ & $6-10$ \\
Visual spatial items (sum score) & & \\
Copy & $10.83 \pm 0.70$ & $8-11$ \\
Delayed recall & $10.7 \pm 2.62$ & $7-14$ \\
Trail making test A & & \\
(time to completion, s) & & \\
Trail making test B & $39.94 \pm 7.07$ & $31-49$ \\
\hline
\end{tabular}

exemplars produced Set $1: 19.38 \pm 6.5$ Set $_{2}: 19.28 \pm 6.4$; Set $\left._{3}: 19.31 \pm 6.2 ; p=0.98\right)$. The three sets were counterbalanced across the group. Prior to scanning, participants were trained using a different set of categories. During scanning, overt responses were recorded using an MRI-compatible microphone and transcribed for subsequent analysis. The scoring of responses was performed by two raters blinded to the stimulation conditions using recorded and transcribed responses. The number of correct responses was determined according to guidelines established during previous studies of our group (Meinzer et al., 2012a, 2013). Repeated measures analysis of variance (RM-ANOVA) assessed performance differences between stimulation conditions.

\section{fMRI DATA ANALYSIS}

Statistical Parametric Mapping (SPM5, Welcome Department of Imaging Neuroscience, London, UK) was used for data analysis. Pre-processing of the data was identical as in our previous studies (Meinzer et al., 2012a, 2013) and comprised re-alignment of functional images, co-registration with the individual participants' anatomical images, unified segmentation and registration to MNI space, and spatial smoothing $\left(8 \times 8 \times 8 \mathrm{~mm}^{3}\right.$ Gaussian kernel). Covariates-of-interest (correct word-retrieval and motor speech baseline condition trials) and movement parameters were included in the design matrix. Afterwards, a high-pass filter (128 s) was applied, data were modelled with a finite impulse response and the contrasts-of-interest were estimated. Those included the comparison of:

1. Correct word-retrieval vs. motor speech trials.

2. Word-retrieval or motor speech trials vs. the implicit baseline as implemented in SPM (Meinzer et al., 2013). The latter were modelled separately to explore potential differential effects of the stimulation on both tasks.
As in our previous study (Lindenberg et al., 2013), we employed an a priori region-of-interest (ROI) approach using the Anatomy Toolbox (Eickhoff et al., 2005). It was previously demonstrated that the semantic word-generation task mainly elicits activity in bilateral frontal cortices in older adults (Meinzer et al., 2009, 2012b,c, 2013), therefore, left and right Brodmann Areas (BA) 44 and 45 were chosen as a priori ROIs to assess the impact of tDCS on language processing. These areas overlap with the anterior (BA45) and posterior (BA44) portions of the inferior frontal gyrus that is tightly connected with the motor system (Eickhoff et al., 2009; Pulvermuller and Fadiga, 2010). These areas also overlapped with regions showing peak activity during the three fMRI sessions in the bilateral frontal cortex. Figure 1C illustrates the activity pattern elicited by the task during the three stimulation conditions, activity patterns surviving a family-wise corrected voxel and cluster threshold of $p<0.05$ are shown. Repeated measures analysis of variance (ANOVA) assessed differences between mean beta activity elicited by the tasks (complex contrast word-retrieval vs. baseline trials; separate comparisons of both tasks vs. the implicit baseline) during the three stimulation conditions in the four frontal ROIs (Greenhouse-Geisser corrected results are reported). Results of post hoc paired $t$-tests were corrected for multiple comparisons using the false discovery rate (FDR; Benjamini and Hochberg, 1995). Pearson correlation coefficients tested whether potential stimulation induced performance improvements would be associated with activity changes in ROIs.

Please note, in response to a reviewer's request during the revision of our previous study (Lindenberg et al., 2013), the impact of the stimulation conditions on activity elicited by the word-retrieval task in M1 (BA4) and premotor (BA6) regions for the complex contrast (word-generation $>$ motor speech baseline condition trials) has already been reported. No activity differences were found between the stimulation conditions. However, as for bilateral frontal ROIs, we also explored whether tDCS would impact selectively on task or baseline trials during the wordgeneration task (Meinzer et al., 2013). This was not the case and we therefore do not report details of this analysis.

\section{RESULTS}

All participants tolerated the stimulation well and no adverse effects were noted. A post-study questionnaire indicated that participants could not differentiate between the stimulation conditions, therefore, effective blinding was achieved by this set-up (for details see Lindenberg et al., 2013).

\section{IMPACT OF tDCS ON PERFORMANCE}

Repeated measures ANOVA revealed significant differences between the three stimulation conditions $\left(F_{(2,16)}=7.74\right.$, $p=0.0004)$. Post hoc paired $t$-tests showed that both active stimulation conditions resulted in superior performance during the semantic word-retrieval task, indicated by a smaller number of errors (anodal-tDCS vs. sham-tDCS: $t_{(17)}=3.37, p=0.0036$, Cohen's $d=0.795$; dual tDCS vs. sham: $t_{(17)}=3.62, p=0.0021$, Cohen's $d=0.853$, Figure 2, both effects remained significant after FDR-correction $p<0.022)$. Performance was comparable in the two active stimulation conditions $(17)=0.33, p=0.74)$. 


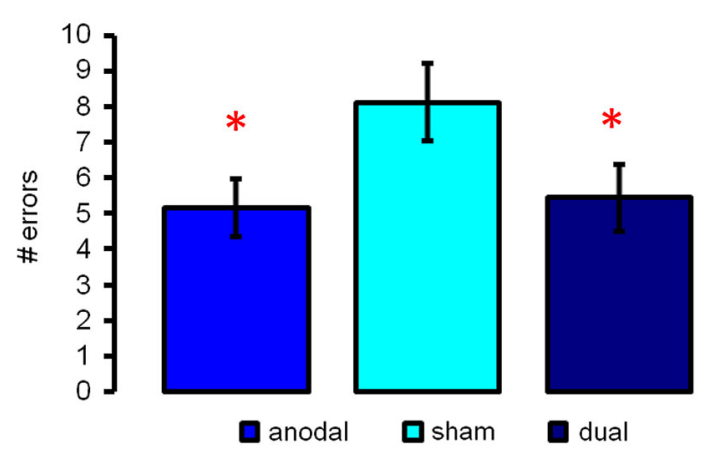

FIGURE 2 | Illustrates semantic word-retrieval performance during the three stimulation conditions (anodal-, sham- and dual-tDCS). Both active stimulation conditions improved performance as compared to sham; no significant differences were found between anodal- and dual-tDCS. Data show mean \pm SEM \# of errors (max. 60$),{ }^{*} p<0.05$

\section{ROI ANALYSIS (WORD-RETRIEVAL VS. MOTOR SPEECH TRIALS, FIGURE 3A)}

Repeated measures ANOVA revealed a significant effect of STIMULATION $\left(F_{(1.2,19.5)}=9.5, p=0.004\right)$. Post hoc paired $t$ tests showed that mean beta activity in both left-sided ROIs was significantly higher during both active stimulation conditions as compared to sham-tDCS (left BA 44/45 anodal-tDCS $>$ sham-tDCS: $t_{(17)}=3.29 / 4.40, p=0.017 / 0.004$; dual-tDCS $>$ sham-tDCS: $\left.t_{(17)}=2.88 / 3.92, p=0.031 / 0.006\right)$. Anodal-tDCS compared to sham-tDCS resulted in increased activity in right BA44 $\left(t_{(17)}=2.52, p=0.044\right)$, the comparison of dual-tDCS vs. sham-tDCS in this ROI did not survive FDR-correction $\left(t_{(17)}=2.29, p=0.06\right)$. However, activity in right BA45 was selectively enhanced during dual-tDCS compared to sham-tDCS $\left(t_{(17)}=2.7, p=0.033\right)$. The main effect of ROI was also significant $\left(F_{(1.6,26.5)}=37.5, p<0.001\right)$, however, this is explained by larger beta values in both left sided ROIs during all stimulation conditions as expected for a left lateralized language task. The interaction STIMULATION $\times$ ROI was not significant $(p=0.439)$. Therefore, active stimulation resulted in enhanced activity in bilateral frontal ROIs. No linear correlations between changed task-related activity and improved performance were found.

\section{ROI ANALYSIS (WORD-RETRIEVAL/MOTOR SPEECH TRIALS VS. IMPLICIT BASELINE, FIGURE 3B)}

Two additional RM-ANOVAs assessed whether word-retrieval or motor speech trials were differentially affected by the respective stimulation conditions. For word-retrieval trials, these analyses revealed no significant effects of STIMULATION $\left(F_{(1.6,27.7)}=1.67, p=0.21\right)$. The interaction of STIMULATION $\times$ ROI approached significance $\left(F_{(2.50,42.6)}=2.95, p=0.052\right)$, however, none of the post hoc comparisons survived an FDRcorrected threshold. Therefore, significant stimulation effects in the comparison above (word-retrieval vs. motor speech trials) were not driven by a direct effect on bilateral frontal activity during word-retrieval trials.

For motor speech trials (Figure 3C), a different pattern emerged. Overall, negative beta values in all ROIs across stimulation conditions indicate that those areas are deactivated during

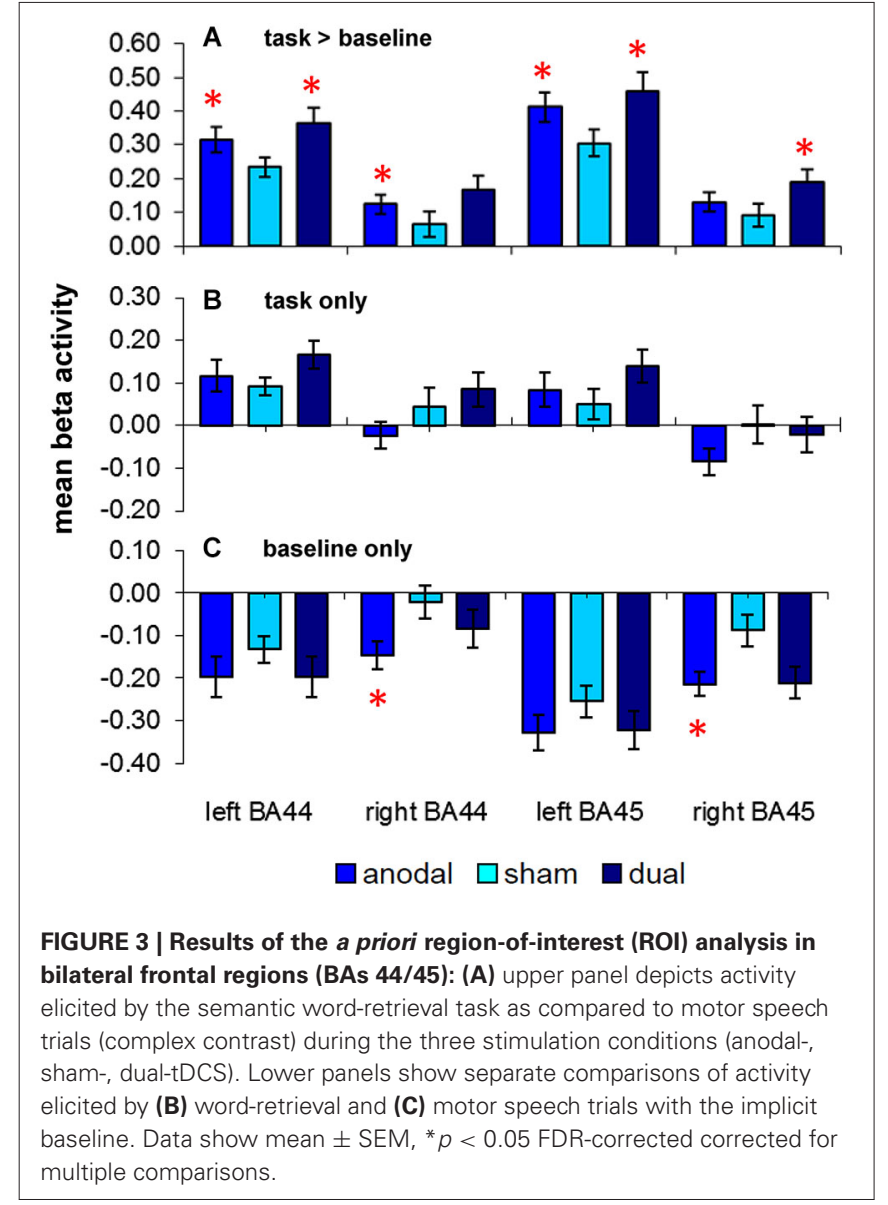

the simple motor speech baseline. Moreover, the degree of deactivation was more pronounced during both active stimulation conditions. Repeated measures ANOVA revealed that the main effect of ROI was significant $\left(F_{(2.2,36.9)}=27.99, p<0.001\right)$ and the effect of STIMULATION approached significance $\left(F_{(1.8,31.5)}=3.26\right.$, $p=0.055)$. Two post hoc comparisons survived an FDR-corrected threshold and revealed a significantly greater decrease of activity during anodal-tDCS compared to sham-tDCS in right BAs 44 and $45\left(t_{(17)}=3.03 / 2.91\right.$, both $\left.p>0.02\right)$. As for the complex contrast (word-retrieval vs. motor speech trials), no linear correlations between changes in activity and performance were found. Despite numerically larger deactivations in all ROIs during dual-tDCS, no significant differences were found compared to sham-tDCS.

In sum, enhanced activity in bilateral frontal ROIs during active-tDCS for the complex contrast (word-retrieval vs. motor speech trials) were mainly explained by more pronounced deactivations in these areas during motor speech trials, while the stimulation did not affect word-retrieval trials.

\section{DISCUSSION}

The results of this proof-of-concept study demonstrate that both anodal and dual-tDCS administered to M1 can improve wordretrieval in healthy older adults. Simultaneous fMRI allowed unprecedented insights into the neural mechanisms mediating tDCS-induced behavioral facilitation. Specifically, improved performance during both active stimulation conditions was 
accompanied by up-regulation of bilateral prefrontal activity as compared to sham-tDCS during the complex contrast of word-retrieval vs. motor speech baseline trials, while no stimulation-effects were found in primary or secondary motor cortices. This effect was mainly driven by more pronounced deactivation of frontal regions during the motor speech baseline task. Given that the human brain operates on limited neural resources (Brem et al., 2014), this may have facilitated switching between the two overlapping neural systems involved in both tasks and in turn explain improved behavioral performance during semantic word-retrieval trials. With regard to clinical applications, the positive behavioral results of this study provide a rationale to explore whether M1 stimulation can enhance treatment outcome in patients with aphasia, thereby providing an exciting novel "backdoor" approach to facilitate language recovery after stroke (Pulvermüller and Berthier, 2008; Meinzer et al., 2011a).

\section{IMPACT OF tDCS ON WORD-RETRIEVAL}

Previous studies that have administered anodal-tDCS to anterior or posterior left-sided perisylvian language regions in healthy individuals reported beneficial stimulation effects during language production (Iyer et al., 2005; Cattaneo et al., 2011; Meinzer et al., 2012a, 2013) and language learning paradigms (de Vries et al., 2010; Meinzer et al., 2014a). Those findings have generated hope that tDCS may be suited to enhance language recovery in patients with post-stroke language impairment. Indeed, a number of studies have provided preliminary evidence that anodal-tDCS administered to perilesional areas in the left hemisphere (Baker et al., 2010; Fridriksson et al., 2011) and also contralesional brain regions (Flöel et al., 2011) may enhance the effectiveness of simultaneous speech therapy. Similarly, dual-tDCS to prefrontal regions enhanced treatment effects in aphasia as well (Marangolo et al., 2013, 2014). However, perilesional tDCS requires a pretreatment fMRI scan to identify residual language cortex in individual patients, which complicates its incorporation in routine clinical practice. Furthermore, up to $40 \%$ of treated patients may not benefit from perilesional stimulation (Baker et al., 2010). Alternative stimulation sites that do not require pre-treatment fMRI scans (e.g., contralesional areas) also yielded variable effects and so far, there is no consensus which areas should be stimulated in individual patients (Meinzer et al., 2011b; Holland and Crinion, 2012). This highlights the need to explore alternative stimulation sites that are easy to implement and also effective in clinical practice.

Stimulation of motor areas that are anatomically and functionally linked to the language system (Dick et al., 2013) may represent an exciting novel approach to facilitate language processing in aphasia (Pulvermüller and Berthier, 2008; Meinzer et al., 2011a). A number of behavioral studies in healthy individuals and patients with aphasia have shown that pre-activation of the motor system can improve language production (Hanlon et al., 1990; Hadar et al., 1998; Holle and Gunter, 2007; Dick et al., 2009; Meinzer et al., 2011a; Benjamin et al., 2014). Moreover, inhibition of M1 by cathodal-tDCS resulted in reduced language learning (Liuzzi et al., 2010). Closer inspection of behavioral improvements reported in aphasia patients after perilesional stimulation in the study by Baker et al. (2010) also revealed that the majority of responsive patients received anodal-tDCS to premotor regions that are tightly connected to M1. In addition, a recent cross-over sham-tDCS controlled single case report reported significantly improved naming ability during anodal-tDCS administered to the left primary motor cortex in a patient with aphasia (Datta et al., 2011). Our results are also in line with recent studies demonstrating that language-motor system interactions may not be limited to action-specific language materials (e.g., verbs describing motor related actions like walking or throwing) but generalize to nonaction related verbs and objects (Liuzzi et al., 2008; Meinzer et al., 2011a; Postle et al., 2013). Therefore, the positive results of our study provide a rationale to assess whether M1 stimulation can enhance treatment outcome in patients with post-stroke aphasia. Given that no major differences were found between anodaland dual-tDCS with regard to performance and brain activity patterns, it is plausible that tDCS effects in the present study are mainly mediated by the left-hemispheric anodal component that was administered in both active stimulation conditions.

\section{IMPACT ON TASK-RELATED ACTIVITY}

A large number of behavioral studies have demonstrated beneficial effects of anodal and dual-tDCS on cognition, language and motor functions (Flöel, 2012, 2014; Monti et al., 2013; Kuo et al., 2014). However, only a handful of studies have combined tDCS with simultaneous fMRI to elucidate the neural underpinnings of these effects (Saiote et al., 2013; Meinzer et al., 2014b). For example, in the motor domain, Antal et al. (2011) demonstrated that anodal-tDCS administered to M1 during a finger tapping task resulted in reduced task-related activity in the pre-supplementary motor area while no effects were found at the stimulation site. Similarly, Lindenberg et al. (2013) did not find activity changes in bilateral M1 after anodal-tDCS administered to the motor cortex, while resting-state fMRI revealed connectivity changes in distant areas, including bilateral prefrontal regions. Therefore, corticospinal excitability changes during M1-tDCS not always translate into changes in regional brain activity changes at the stimulation site as measured by fMRI but may affect functionally connected remote brain regions. Similarly, M1 stimulation did not impact on task-related activity in primary and secondary motor cortices during the word-retrieval task in the present study, however, stimulation effects were found in functionally connected bilateral IFG (Eickhoff et al., 2009).

Previous studies that employed word-retrieval tasks during anodal-tDCS of left perisylvian regions improved picture naming and semantic word-generation performance in healthy older adults which was associated with reduced task-related activity in left-sided (Holland et al., 2011; Meinzer et al., 2012a) or bilateral IFG (Meinzer et al., 2013). In those studies, reduced activity was interpreted as more efficient processing due to tDCSinduced neural facilitation. Moreover, using the same task as in the present study, this effect could be explained by selectively reduced activity during word-retrieval trials, but not during simple motor speech trials (Meinzer et al., 2013). In the present study, we show that improved word-retrieval after M1-tDCS was mediated by a different mechanism. Specifically, active-tDCS did not impact on task-related activity during word-retrieval trials, but resulted in more pronounced deactivation of bilateral prefrontal 
activity during motor speech trials, which explains the net activity increase in those areas, emphasizing specific tDCS-effects on different tasks (Iuculano and Cohen Kadosh, 2013). Compared to sham-tDCS, the degree of prefrontal deactivation was numerically similar during both active stimulation conditions; however, it only reached significance in right-frontal regions during anodaltDCS. Tentatively, this finding could be explained by higher current flow to right frontal regions during unilateral M1-tDCS with a supraorbital reference electrode as suggested by previous modelling studies (Wagner et al., 2007; Bikson et al., 2012; Kuo et al., 2013).

Funnelling of information that arises from different neural networks during dual task performance in the prefrontal cortex has been suggested to create a bottleneck for information processing and consequently lower behavioral output. Training can improve dual-task performance by inducing more efficient neural processing, typically expressed as reduced activity during fMRI in prefrontal cortex (Dux et al., 2009). Similarly, behavioral improvement in the present study may have been mediated by further disengagement (deactivation) of prefrontal regions during motor speech trials, possibly freeing processing resources or reducing switching costs between the two tasks that are engaging partially overlapping networks (Brem et al., 2014). However, this hypothesis needs to be scrutinized in future studies that allow examining interactions between the neural networks for speech production and lexical retrieval and their modulation by tDCS using different imaging paradigms and functional connectivity analysis. Such an analysis was not feasible in this present study due to the blocked-sparse sampling design. Moreover, brain regions in the vicinity of the targeted M1 may also undergo excitability changes due to the large size of the electrode or spill-over effects (Bestmann et al., 2004) and it is conceivable that stimulation effects may have extended into the neighboring parietal areas. Future studies are thus required to determine whether prefrontal activity changes during M1 stimulation are mediated by direct effects of M1 on interconnected prefrontal regions or by modulation of a larger fronto-parietal network.

\section{CONCLUSIONS}

In sum, the present study demonstrates that M1 stimulation can improve word-retrieval in healthy older individuals and provides first evidence for the underlying neural mechanisms mediating behavioral facilitation. Our results also provide a rationale to explore the effectiveness of M1 stimulation as an alternative and clinically feasible adjunct treatment approach in poststroke aphasia. In addition, our findings confirm that languagemotor interactions may extend beyond the well-known impact on action-specific language material (Liuzzi et al., 2008; Meinzer et al., 2011a; Postle et al., 2013), which emphasizes its broad potential to enhance language recovery in clinical settings. Finally, the imaging results emphasize that different tDCS-montages may result in specific neural effects on different tasks that require further scrutiny.

\section{ACKNOWLEDGMENTS}

This work was supported by grants from the Australian Research Council (Marcus Meinzer: ARC FT120100608), the Deutsche
Forschungsgemeinschaft (Agnes Flöel: 379-8/1; 379-10/1, 37911/1, DFG-Exc-257, Lena Ulm: 423/1-1), the Bundesministerium für Bildung und Forschung (Agnes Flöel: FKZ0315673A and 01GY1144; Agnes Flöel and Marcus Meinzer: 01EO0801), the Else-Kröner Fresenius Stiftung (Robert Lindenberg: 2011-119).

\section{REFERENCES}

Antal, A., Polania, R., Schmidt-Samoa, C., Dechent, P., and Paulus, W. (2011). Transcranial direct current stimulation over the primary motor cortex during fMRI. Neuroimage 55, 590-596. doi: 10.1016/j.neuroimage.2010.11.085

Baker, J. M., Rorden, C., and Fridriksson, J. (2010). Using transcranial directcurrent stimulation to treat stroke patients with aphasia. Stroke 41, 1229-1236. doi: 10.1161/STROKEAHA.109.576785

Behrens, T. E., and Sporns, O. (2012). Human connectomics. Curr. Opin. Neurobiol. 22, 144-153. doi: 10.1016/j.conb.2011.08.005

Benjamin, M. L., Towler, S., Garcia, A., Park, H., Sudhyadhom, A., Harnish, S., et al. (2014). A behavioral manipulation engages right frontal cortex during aphasia therapy. Neurorehabil. Neural Repair 28, 545-553. doi: 10. $1177 / 1545968313517754$

Benjamini, Y., and Hochberg, Y. (1995). Controlling the false discovery rate: a practical and powerful approach to multiple testing. J. R. Statist. Soc. B 57, 289-300.

Bestmann, S., Baudewig, J., Siebner, H. R., Rothwell, J. C., and Frahm, J. (2004). Functional MRI of the immediate impact of transcranial magnetic stimulation on cortical and subcortical motor circuits. Eur. J. Neurosci. 19, 1950-1962. doi: 10.1111/j.1460-9568.2004.03277.x

Bikson, M., Rahman, A., Datta, A., Fregni, F., and Merabet, L. (2012). Highresolution modeling assisted design of customized and individualized transcranial direct current stimulation protocols. Neuromodulation 15, 306-315. doi: 10. 1111/j.1525-1403.2012.00481.x

Brem, A. K., Fried, P. J., Horvath, J. C., Robertson, E. M., and Pascual-Leone, A. (2014). Is neuroenhancement by noninvasive brain stimulation a net zero-sum proposition? Neuroimage 85(Pt. 3), 1058-1068. doi: 10.1016/j.neuroimage.2013. 07.038

Bressler, S. L., and Menon, V. (2010). Large-scale brain networks in cognition: emerging methods and principles. Trends Cogn. Sci. 14, 277-290. doi: 10.1016/j. tics.2010.04.004

Cattaneo, Z., Pisoni, A., and Papagno, C. (2011). Transcranial direct current stimulation over Broca's region improves phonemic and semantic fluency in healthy individuals. Neuroscience 183, 64-70. doi: 10.1016/j.neuroscience.2011. 03.058

Crosson, B. (2013). Thalamic mechanisms in language: a reconsideration based on recent findings and concepts. Brain Lang. 126, 73-88. doi: 10.1016/j.bandl.2012. 06.011

Datta, A., Baker, J. M., Bikson, M., and Fridriksson, J. (2011). Individualized model predicts brain current flow during transcranial direct-current stimulation treatment in responsive stroke patient. Brain Stimul. 4, 169-174. doi: 10.1016/j. brs.2010.11.001

de Vries, M. H., Barth, A. C., Maiworm, S., Knecht, S., Zwitserlood, P., and Flöel, A. (2010). Electrical stimulation of Broca's area enhances implicit learning of an artificial grammar. J. Cogn. Neurosci. 22, 2427-2436. doi: 10.1162/jocn.2009. 21385

Dick, A. S., Bernal, B., and Tremblay, P. (2013). The language connectome: new pathways, new concepts. Neuroscientist doi: 10.1177/1073858413513502. [Epub ahead of print].

Dick, A. S., Goldin-Meadow, S., Hasson, U., Skipper, J. I., and Small, S. L. (2009). Co-speech gestures influence neural activity in brain regions associated with processing semantic information. Hum. Brain Mapp. 30, 3509-3526. doi: 10. 1002/hbm.20774

Dux, P. E., Tombu, M. N., Harrison, S., Rogers, B. P., Tong, F., and Marois, R. (2009). Training improves multitasking performance by increasing the speed of information processing in human prefrontal cortex. Neuron 63, 127-138. doi: 10.1016/j.neuron.2009.06.005

Eickhoff, S. B., Heim, S., Zilles, K., and Amunts, K. (2009). A systems perspective on the effective connectivity of overt speech production. Philos. Trans. A Math. Phys. Eng. Sci. 367, 2399-2421. doi: 10.1098/rsta.2008.0287

Eickhoff, S. B., Stephan, K. E., Mohlberg, H., Grefkes, C., Fink, G. R., Amunts, K., et al. (2005). A new SPM toolbox for combining probabilistic cytoarchitectonic 
maps and functional imaging data. Neuroimage 25, 1325-1335. doi: 10.1016/j. neuroimage.2004.12.034

Flöel, A. (2012). Non-invasive brain stimulation and language processing in the healthy brain. Aphasiology 26, 1082-1102. doi: 10.1080/02687038.2011. 589892

Flöel, A. (2014). tDCS-enhanced motor and cognitive function in neurological diseases. Neuroimage 85(Pt. 3), 934-947. doi: 10.1016/j.neuroimage.2013. 05.098

Flöel, A., Meinzer, M., Kirstein, R., Nijhof, S., Deppe, M., Knecht, S., et al. (2011). Short-term anomia training and electrical brain stimulation. Stroke 42, 2065 2067. doi: 10.1161/STROKEAHA.110.609032

Fridriksson, J., Richardson, J. D., Baker, J. M., and Rorden, C. (2011). Transcranial direct current stimulation improves naming reaction time in fluent aphasia: a double-blind, sham-controlled study. Stroke 42, 819-821. doi: 10. 1161/STROKEAHA.110.600288

Hadar, U., Wenkert-Olenik, D., Krauss, R., and Soroker, N. (1998). Gesture and the processing of speech: neuropsychological evidence. Brain Lang. 62, 107-126. doi: 10.1006/brln.1997.1890

Hanlon, R. E., Brown, J. W., and Gerstman, L. J. (1990). Enhancement of naming in nonfluent aphasia through gesture. Brain Lang. 38, 298-314. doi: 10.1016/0093934x(90)90116-x

Harnish, S., Meinzer, M., Trinastic, J., Fitzgerald, D., and Page, S. (2011). Language changes coincide with motor and fMRI changes following upper extremity motor therapy for hemiparesis: a brief report. Brain Imaging Behav. doi: 10 . 1007/s11682-011-9139-y. [Epub ahead of print].

Holland, R., and Crinion, J. (2012). Can tDCS enhance treatment of aphasia after stroke? Aphasiology 26, 1169-1191. doi: 10.1080/02687038.2011.616925

Holland, R., Leff, A. P., Josephs, O., Galea, J. M., Desikan, M., Price, C. J., et al. (2011). Speech facilitation by left inferior frontal cortex stimulation. Curr. Biol. 21, 1403-1407. doi: 10.1016/j.cub.2011.07.021

Holle, H., and Gunter, T. C. (2007). The role of iconic gestures in speech disambiguation: ERP evidence. J. Cogn. Neurosci. 19, 1175-1192. doi: 10.1162/jocn. 2007.19.7.1175

Iuculano, T., and Cohen Kadosh, R. (2013). The mental cost of cognitive enhancement. J. Neurosci. 33, 4482-4486. doi: 10.1523/JNEUROSCI.4927-12.2013

Iyer, M. B., Mattu, U., Grafman, J., Lomarev, M., Sato, S., and Wassermann, E. M. (2005). Safety and cognitive effect of frontal DC brain polarization in healthy individuals. Neurology 64, 872-875. doi: 10.1212/01.wnl.0000152986. 07469.e9

Kuo, H. I., Bikson, M., Datta, A., Minhas, P., Paulus, W., Kuo, M. F., et al. (2013). Comparing cortical plasticity induced by conventional and high-definition $4 \times 1$ ring tDCS: a neurophysiological study. Brain Stimul. 6, 644-648. doi: 10.1016/j. brs.2012.09.010

Kuo, M. F., Paulus, W., and Nitsche, M. A. (2014). Therapeutic effects of noninvasive brain stimulation with direct currents (tDCS) in neuropsychiatric diseases. Neuroimage 85(Pt. 3), 948-960. doi: 10.1016/j.neuroimage.2013.05.117

Lindenberg, R., Nachtigall, L., Meinzer, M., Sieg, M. M., and Flöel, A. (2013). Differential effects of dual and unihemispheric motor cortex stimulation in older adults. J. Neurosci. 33, 9176-9183. doi: 10.1523/JNEUROSCI.0055-13. 2013

Lindenberg, R., Renga, V., Zhu, L. L., Nair, D., and Schlaug, G. (2010). Bihemispheric brain stimulation facilitates motor recovery in chronic stroke patients. Neurology 75, 2176-2184. doi: 10.1212/WNL.0b013e318202013a

Lindenberg, R., Zhu, L. L., and Schlaug, G. (2012). Combined central and peripheral stimulation to facilitate motor recovery after stroke: the effect of number of sessions on outcome. Neurorehabil. Neural Repair 26, 479-483. doi: 10. $1177 / 1545968311427568$

Liuzzi, G., Ellger, T., Flöel, A., Breitenstein, C., Jansen, A., and Knecht, S. (2008). Walking the talk—speech activates the leg motor cortex. Neuropsychologia 46, 2824-2830. doi: 10.1016/j.neuropsychologia.2008.05.015

Liuzzi, G., Freundlieb, N., Ridder, V., Hoppe, J., Heise, K., Zimerman, M., et al. (2010). The involvement of the left motor cortex in learning of a novel action word lexicon. Curr. Biol. 20, 1745-1751. doi: 10.1016/j.cub.2010.08.034

Mannhaupt, H. R. (1983). Produktionsnormen für verbale Reaktionen zu 40 geläufigen Kategorien. Lang. Cogn. 4, 264-278.

Marangolo, P., Fiori, V., Cipollari, S., Campana, S., Razzano, C., Di Paola, M., et al. (2013). Bihemispheric stimulation over left and right inferior frontal region enhances recovery from apraxia of speech in chronic aphasia. Eur. J. Neurosci. 38, 3370-3377. doi: 10.1111/ejn.12332
Marangolo, P., Fiori, V., Gelfo, F., Shofany, J., Razzano, C., Caltagirone, C., et al. (2014). Bihemispheric tDCS enhances language recovery but does not alter BDNF levels in chronic aphasic patients. Restor. Neurol. Neurosci. 32, 367-379. doi: 10.3233/RNN-130323

Meinzer, M., Antonenko, D., Lindenberg, R., Hetzer, S., Ulm, L., Avirame, K., et al. (2012a). Electrical brain stimulation improves cognitive performance by modulating functional connectivity and task-specific activation. J. Neurosci. 32, 1859-1866. doi: 10.1523/JNEUROSCI.4812-11.2012

Meinzer, M., Breitenstein, C., Westerhoff, U., Sommer, J., Rösser, N., Rodriguez, A. D., et al. (2011a). Motor cortex preactivation by standing facilitates word retrieval in aphasia. Neurorehabil. Neural Repair 25, 178-187. doi: 10. $1177 / 1545968310376577$

Meinzer, M., Flaisch, T., Seeds, L., Harnish, S., Antonenko, D., Witte, V., et al. (2012b). Same modulation but different starting points: performance modulates age differences in inferior frontal cortex activity during word-retrieval. PLoS One 7:e33631. doi: 10.1371/journal.pone.0033631

Meinzer, M., Flaisch, T., Wilser, L., Eulitz, C., Rockstroh, B., Conway, T., et al. (2009). Neural signatures of semantic and phonemic fluency in young and old adults. J. Cogn. Neurosci. 21, 2007-2018. doi: 10.1162/jocn.2009. 21219

Meinzer, M., Harnish, S., Conway, T., and Crosson, B. (2011b). Recent developments in functional and structural imaging of aphasia recovery after stroke. Aphasiology 25, 271-290. doi: 10.1080/02687038.2010.530672

Meinzer, M., Jähnigen, S., Copland, D. A., Darkow, R., Grittner, U., Avirame, K., et al. (2014a). Transcranial direct current stimulation over multiple days improves learning and maintenance of a novel vocabulary. Cortex 50, 137-147. doi: 10.1016/j.cortex.2013.07.013

Meinzer, M., Lindenberg, R., Antonenko, D., Flaisch, T., and Flöel, A. (2013). Anodal transcranial direct current stimulation temporarily reverses ageassociated cognitive decline and functional brain activity changes. J. Neurosci. 33, 12470-12478. doi: 10.1523/JNEUROSCI.5743-12.2013

Meinzer, M., Lindenberg, R., Darkow, R., Ulm, L., Copland, D., and Flöel, A. (2014b). Transcranial direct current stimulation and simultaneous functional magnetic resonance imaging. J. Vis. Exp., e51730. doi: 10.3791/51730

Meinzer, M., Seeds, L., Flaisch, T., Harnish, S., Cohen, M. L., McGregor, K., et al. (2012c). Impact of changed positive and negative task-related brain activity on word-retrieval in aging. Neurobiol. Aging 33, 656-669. doi: 10.1016/j. neurobiolaging.2010.06.020

Miniussi, C., Harris, J. A., and Ruzzoli, M. (2013). Modelling non-invasive brain stimulation in cognitive neuroscience. Neurosci. Biobehav. Rev. 37, 1702-1712. doi: 10.1016/j.neubiorev.2013.06.014

Monti, A., Ferrucci, R., Fumagalli, M., Mameli, F., Cogiamanian, F., Ardolino, G., et al. (2013). Transcranial direct current stimulation (tDCS) and language. J. Neurol. Neurosurg. Psychiatry 84, 832-842. doi: 10.1136/jnnp-2012302825

Oldfield, R. C. (1971). The assessment and analysis of handedness: the Edinburgh inventory. Neuropsychologia 9, 97-113. doi: 10.1016/0028-3932(71)90067-4

Pine, K. J., Bird, H., and Kirk, E. (2007). The effects of prohibiting gestures on children's lexical retrieval ability. Dev. Sci. 10, 747-754. doi: 10.1111/j.14677687.2007.00610.x

Pobric, G., and Hamilton, A. F. (2006). Action understanding requires the left inferior frontal cortex. Curr. Biol. 16, 524-529. doi: 10.1016/j.cub.2006. 01.033

Postle, N., Ashton, R., McFarland, K., and de Zubicaray, G. I. (2013). No specific role for the manual motor system in processing the meanings of words related to the hand. Front. Hum. Neurosci. 7:11. doi: 10.3389/fnhum.2013. 00011

Pulvermüller, F., and Berthier, M. L. (2008). Aphasia therapy on a neuroscience basis. Aphasiology 22, 563-599. doi: 10.1080/02687030701612213

Pulvermuller, F., and Fadiga, L. (2010). Active perception: sensorimotor circuits as a cortical basis for language. Nat. Rev. Neurosci. 11, 351-360. doi: 10. 1038/nrn2811

Rauscher, F. H., Krauss, R. M., and Chen, Y. (1996). Gesture, speech, and lexical access: the role of lexical movements in speech production. Psychol. Sci. 7, 226231. doi: 10.1111/j.1467-9280.1996.tb00364.x

Reis, J., Schambra, H. M., Cohen, L. G., Buch, E. R., Fritsch, B., Zarahn, E., et al. (2009). Noninvasive cortical stimulation enhances motor skill acquisition over multiple days through an effect on consolidation. Proc. Natl. Acad. Sci. U S A 106, 1590-1595. doi: 10.1073/pnas.0805413106 
Rizzolatti, G., and Craighero, L. (2004). The mirror-neuron system. Ann. Rev. Neurosci. 27, 169-192. doi: 10.1146/annurev.neuro.27.070203.144230

Saiote, C., Turi, Z., Paulus, W., and Antal, A. (2013). Combining functional magnetic resonance imaging with transcranial electrical stimulation. Front. Hum. Neurosci. 7:435. doi: 10.3389/fnhum.2013.00435

Stagg, C. J., and Nitsche, M. A. (2011). Physiological basis of transcranial direct current stimulation. Neuroscientist 17, 37-53. doi: 10.1177/10738584103 86614

Thompson-Schill, S. L., D’Esposito, M., Aguirre, G. K., and Farah, M. J. (1997). Role of left inferior prefrontal cortex in retrieval of semantic knowledge: a reevaluation. Proc. Natl. Acad. Sci. U S A 94, 14792-14797. doi: 10.1073/pnas. 94.26.14792

Vines, B. W., Cerruti, C., and Schlaug, G. (2008). Dual-hemisphere tDCS facilitates greater improvements for healthy subjects' non-dominant hand compared to uni-hemisphere stimulation. BMC Neurosci. 9:103. doi: 10.1186/1471-22029-103

Wagner, T., Fregni, F., Fecteau, S., Grodzinsky, A., Zahn, M., and Pascual-Leone, A. (2007). Transcranial direct current stimulation: a computer-based human model study. Neuroimage 35, 1113-1124. doi: 10.1016/j.neuroimage.2007. 01.027
Willems, R. M., and Hagoort, P. (2007). Neural evidence for the interplay between language, gesture and action: a review. Brain Lang. 101, 278-289. doi: 10.1016/j. bandl.2007.03.004

Conflict of Interest Statement: The authors declare that the research was conducted in the absence of any commercial or financial relationships that could be construed as a potential conflict of interest.

Received: 15 June 2014; accepted: 06 September 2014; published online: 23 September 2014.

Citation: Meinzer M, Lindenberg R, Sieg MM, Nachtigall L, Ulm L and Flöel A (2014) Transcranial direct current stimulation of the primary motor cortex improves wordretrieval in older adults. Front. Aging Neurosci. 6:253. doi: 10.3389/fnagi.2014.00253 This article was submitted to the journal Frontiers in Aging Neuroscience.

Copyright (c) 2014 Meinzer, Lindenberg, Sieg, Nachtigall, Ulm and Flöel. This is an open-access article distributed under the terms of the Creative Commons Attribution License (CC BY). The use, distribution and reproduction in other forums is permitted, provided the original author(s) or licensor are credited and that the original publication in this journal is cited, in accordance with accepted academic practice. No use, distribution or reproduction is permitted which does not comply with these terms. 